\title{
¿Por qué la Unión Europea debería ser de relevancia para América Latina y el Caribe?
}

Alberto Manuel Poletti Adorno*

\section{UE-LAC: UNA RELACIÓN CON VARIOS FRENTES}

La utilización de ejemplos en el marco de una familia para mostrar las diferencias no siempre es del agrado de todos. Algunos padres comparan a sus hijos y esas comparaciones son odiosas. No obstante, tomamos el riesgo de realizar comparaciones entre la Unión Europea (UE) y la región de América Latina y el Caribe (ALC) y para ello, dejaremos de lado un momento los vínculos históricos, las migraciones y los intercambios económicos para centrarnos en la experiencia frente a la resolución de problemas internos que enfrentan los Estados miembros de la UE y los países de ALC.

Y es allí donde la práctica en la construcción del Estado de Derecho sin duda se encuentra mucho más avanzada en los países de la UE. Nótese que dentro de los criterios de Copenhague para la adhesión ${ }^{1}$, se menciona justamente este requisito. Mientras que en América se toman más que nada criterios geográficos o ideológicos para decidir una asociación.

Y pese a ello, no es ocioso mencionar que los países de América Latina han avanzado si los comparamos con las épocas de la Dictadura. Hoy en día cuentan con un sistema regional de defensa de derechos humanos que, aunque con sus propios problemas económicos y retrasos, funciona. También se ha avanzado en el ámbito económico, pero persisten las desigualdades sociales. La justicia en muchos países está bajo la lupa y las instituciones no funcionan como deberían para alcanzar el grado de un Estado desarrollado.

\footnotetext{
* Abogado egresado de la Universidad Nacional de Asunción, Paraguay. Doctor en Derecho de la Universidad París 1 Panthéon-Sorbonne. Profesor de derecho internacional de la Universidad Columbia del Paraguay. Investigador externo del Dpto. de Derecho Político de la UNED, España. Analista Senior del Digesto Legislativo del CEDEP. Las opiniones descritas corresponden exclusivamente al autor y no comprometen a las instituciones mencionadas. Correo-e: ap@polettiadorno.com
} 
Ian Bremmer es un profesor norteamericano que al analizar el ascenso y evolución de los países, explicó el sistema de la "Curva J" que analiza el grado de estabilidad y desarrollo/apertura de los Estados'. De hecho señala a muchos de los países de la UE en la parte más alta. En cuanto a América Latina, muchos Estados cuentan aún con dificultades para llegar a esa posición. Es entonces cuando nos preguntamos que experiencias europeas podrían ser útiles para el mejoramiento de los sistemas latinoamericanos y del Caribe.

\section{LA DEFENSA DEL ESTADO DE DERECHO COMO MECANISMO PARA AVANZAR EN LA ASOCIACIÓN ENTRE PAÍSES}

Se ha mencionado que la posición de business as usual ya no es viable y que para avanzar en procesos de integración, los países deben contar con regímenes que permitan generar confianza ${ }^{3}$. Más allá de las inversiones existentes, las falencias en numerosos ámbitos en LAC no permiten que muchos inversionistas europeos puedan decidir la colación de sus capitales en la región LAC.

Pero fuera de ello, la experiencia de América Latina en algunos ámbitos también puede ser de interés para la contraparte europea. Nótese que más allá de las oportunidades en un continente joven y con recursos han surgido grandes exponentes en todos los ámbitos de la ciencia, la cultura, el deporte y el arte por no citar sino algunas ramas del conocimiento. Por ende, no se trata de avanzar en una relación en la que solo de una de las partes aporte conocimientos y experiencias, sino que ambas partes sigan siendo beneficiadas, aprendiendo de la otra, compartiendo experiencias y conocimientos.

Por ende, en este trabajo nos centraremos en las experiencias para avanzar en el Estado de Derecho y el compromiso de compartir experiencias positivas.

\section{LA PERSPECTIVA DE COLABORACIÓN EN LA OBTENCIÓN DE LOS OBJETIVOS DE DESARROLLO SOSTENIBLE 2030}

Para muchos países de LAC con perspectivas de desarrollo es importante tomar en cuenta las legislaciones y experiencias en la solución de problemas vinculados al sistema de justicia, promoción y vigencia de las instituciones públicas o simplemente para contar con mecanismos que permitan hacer uso de los derechos.

En el ámbito jurídico si la gran mayoría de los países iberoamericanos han seguido el sistema de derecho continental, no cabe duda que las naciones del Caribe han optado por el sistema del common law. Resulta importante mencionar que el CARICOM ha creado una corte de justicia que, además de tener competencia para juzgar el proceso de integración, tiene igualmente competencia para decidir en última instancia varios asuntos internos de los países ${ }^{4}$, reemplazando a los tribunales ingleses. En el UNASUR se ha pensado también en crear una corte 
penal suramericana ${ }^{5}$. Estos ejemplos nos permiten señalar que más allá de las críticas que puedan válidamente hacerse a algunos aspectos de la integración regional, persiste la creencia de que existen problemas comunes entre los países a los que se brindará juntos una mejor respuesta.

Esta interacción y la discusión de casos resueltos, explicando la diferencia de jurisprudencia o la conformidad de soluciones permitirá a los diferentes sectores sociales explicar con bases sólidas las diferencias que existen entre los países a ambos lados del océano Atlántico.

\section{UNA AGENDA COMÚN Y NUMEROSOS EJEMPLOS DE COOPERACIÓN}

Los problemas comunes para los habitantes del planeta tales como la lucha contra el cambio climático, el terrorismo o la seguridad ciudadana conllevan esfuerzos de ciudadanos, asociaciones y gobiernos de todos los países.

Se ha mencionado que existen más de 1300 programas apoyados en LAC para la protección del medio ambiente y que desde 1994 la UE ha contribuido con más de 1.000 millones de Euros a la cooperación regional con América Latina a través de distintos programas de cooperación ${ }^{6}$.

El eurodiputado español Xavi López ha expuesto con relación al informe aprobado en la comisión de Asuntos Exteriores del Parlamento Europeo sobre las nuevas relaciones políticas con América Latina que "con connotaciones diferentes, hemos padecido directamente durante los últimos años el terrorismo. América Latina tiene problemas derivados del narcotráfico y el crimen organizado. Es verdad que son retos diferentes bajo el rubro de la seguridad. Podemos compartir información, compartir acción para luchar desde el terrorismo hasta el crimen organizado"?

Debido a las cercanías descritas más arriba, la cooperación UE-LAC se encuentra facilitada. De hecho, millones de personas han cruzado el océano desde el descubrimiento del nuevo rumbo en ambas direcciones. Esta realidad hace vayan incrementándose los intercambios y que algunas ideas vayan desarrollándose en ambos lados.

\subsection{LA ASISTENCIA CONSULAR: UNA REFERENCIA IMPORTANTE}

Los Estados miembros de la Unión europea establecieron ya en el año 1995 medidas para la protección de ciudadanos europeos fuera del continente por Decisión 95/553/CE, luego incluyeron las medidas en los artículos 20 y 23 
del Tratado de Lisboa y en el art. 46 de la Carta de Derechos fundamentales de la UE se reconoció el derecho a la asistencia consular. Finalmente adoptaron la Directiva (UE) 2015/637 del 20 de abril de 2015 sobre las medidas de coordinación y cooperación para facilitar la protección consular de ciudadanos de la Unión no representados en terceros países ${ }^{8}$.

En el MERCOSUR, por Decisión del Consejo Mercado Común $\mathbf{N}^{\circ}$ 35/00 del 29 de junio de 2000 se estableció el Mecanismo de cooperación consular entre los países del MERCOSUR, Bolivia y Chile. Conforme al art. 3 de la citada norma, inicialmente, se definieron como materia de dicha cooperación consular las siguientes acciones:

- Permitir la utilización de la dirección postal de la Oficina Consular para la recepción de la correspondencia privada.

- Orientar en materia de asistencia médica y jurídica en la jurisdicción.

- Brindar asistencia humanitaria a personas accidentadas o en situaciones de emergencia e informar al respecto, a través de la oficina de Asuntos Consulares de la Cancillería del país de origen del recurrente, como canal diplomático competente.

- Informar a parientes o personas allegadas sobre accidentes, fallecimientos o catástrofes, a través de los mismos canales diplomáticos.

- Interesarse por los nacionales de los países del MERCOSUR, Bolivia y Chile detenidos o en prisión, comunicando la situación a su país de origen a través de los canales diplomáticos.

- Procurar la localización de personas en la jurisdicción y transmitir la información al país de origen a través de los canales diplomáticos.

La Comunidad Andina también aprobó un mecanismo de cooperación consular ${ }^{9}$ entre los Estados miembros en febrero de 2013.

Es importante destacar que los países de la Alianza del Pacífico también adoptaron disposiciones similares ${ }^{10}$ en la VIII Cumbre de la Alianza celebrada en Cartagena, Colombia en febrero de 2014.

¿Podría pensarse en un mecanismo de cooperación consular UE-LAC? Más allá de que las relaciones entre los países son buenas y que la cooperación se da en forma adecuada, es importante destacar que este ejemplo, llevado a otros ámbitos, podría ser un importante ejemplo de la necesidad de cooperación y relevancia entre ambos bloques de países.

En estos momentos, pensar en una asociación con los Estados Unidos (país con el que varias naciones de América Latina tienen mayor afinidad) resulta poco probable. Sin embargo, avanzar en una cooperación entre los Estados UELAC si resulta factible. De hecho, se sigue discutiendo la creación de una zona común de libre comercio y se avanzan hacia otros desafíos. 


\subsection{LA DEFENSA DE LOS DERECHOS FUNDAMENTALES}

Es imposible dejar de mencionar que luego de la Carta de Bogotá ${ }^{11}$ donde se hizo referencia a la Comisión interamericana de derechos Humanos, en Europa se suscribió el Convenio para la protección de los derechos humanos y de las libertades fundamentales ${ }^{12}$ suscrito en Roma del 4 de noviembre de 1950 que derivó en un amplio sistema de defensa derechos.

La interacción de los tribunales europeo e interamericano de derechos fundamentales se notable. Varios de los casos resueltos en un continente fueron posteriormente adoptados o han servido de base para la discusión de temas similares en otro lugar.

Más allá de los numerosos ejemplos sobre lo expuesto que pueden surgir de la lectura de la jurisprudencia de los tribunales internacionales, es importante destacar que el sistema no está exento de críticas y muchos se preguntan si, ante la vigencia de los organismos del sistema de naciones unidas de derechos humanos, resulta oportuno continuar con los organismos regionales habida cuenta que en algunas regiones (piénsese en la región Asia-Pacífico) no existen organismos regionales.

Creemos firmemente que la existencia de estos organismos requiere ser potenciada para que las personas puedan acceder en mejores condiciones a organismos cuyos derechos no son siempre reconocidos a nivel interno.

\subsection{LOS CONFLICTOS EN MATERIA DE NACIONALIDAD}

Los sistemas de atribución de nacionalidad jus solis y jus sanguinis prevalecen en el continente americano y europeo. Al ser este aspecto un tema que vincula a la soberanía los estados no se ha discutido aún un consenso en la materia, pero se han firmado numerosos tratados en la materia. Así, la Convención de 1954 sobre el Estatuto de los apátridas ${ }^{13}$ y la Convención de 1961 para evitar los casos de apatridia ${ }^{14}$. Ahora bien, si muchos países latinoamericanos han suscrito estos tratados, los Estados europeos aún no han tomado la decisión de hacerlo.

La apatridia afecta a cerca de 10.000 .000 en el mundo y las deja sin protección ${ }^{15}$ afectando especialmente a niños hijos de migrantes de algunos países de América Latina a Europa que no pueden acceder a la nacionalidad del país de nacimiento y que los Estados de origen de los padres no prevén la transmisión automática de la nacionalidad sin residencia en el país de los mismos ${ }^{16}$.

Mucho se ha discutido también sobre la doble o múltiple nacionalidad, un realidad vinculada a la confluencia en una persona de dos o más vínculos con más de un Estado. Así, podrá verse que si en Europa se ha firmado un Convenio en la materia ${ }^{17}$, en América latina el tema no ha sido aún objeto de atención. 


\subsection{LA LUCHA CONTRA LA MIGRACIÓN CLANDESTINA}

Algunos países de América que adoptaron sus legislaciones en el Siglo XIX veían con buenos ojos la migración. El artículo 25 de la Constitución argentina de 1853 dispone: "El Gobierno federal fomentará la inmigración europea; y no podrá restringir, limitar ni gravar con impuesto alguno la entrada en el territorio argentino de los extranjeros que traigan por objeto labrar la tierra, mejorar las industrias, e introducir y enseñar las ciencias y las artes" ${ }^{18}$. En términos similares se redactó el artículo 6 de la Constitución de Paraguay de 1870.

Las migraciones existieron desde la antigüedad. Durante el Siglo XIX, muchos europeos se dirigieron hacia América por razones económicas. Pero a inicios del siglo XXI, el camino inverso fue el escogido. Y también se siguieron produciendo migraciones dentro de ambos continentes.

En el caso de Centroamérica, miles de niños y jóvenes de El Salvador, Honduras y Guatemala se ven forzados a abandonar sus viviendas por la amenaza de bandas criminales. Solo en estos tres países hay más de 7 millones de personas desplazadas, según la ONU ${ }^{19}$. También muchos peruanos y colombianos han migrado en las últimas décadas del siglo pasado debido a conflictos armados en sus países, habiendo muchos de ellos retornado al mejorar la seguridad. Hoy se hace referencia a la diáspora haitiana, a la venezolana y sin hacer referencia a otros países, el control de la migración se hace necesario frente a peligrosas rutas empleadas por personas que huyen de sus realidades en la búsqueda de un futuro pero que, debido a la peligrosidad de los medios empleados, corren el riesgo de fallecer ${ }^{20}$.

En Europa, la migración proveniente no solo de países como Libia, Siria y otros Estados con serios problemas económicos y de seguridad hacen que en muchos Estados se hayan levantado voces contra los migrantes que incluso ocupan cargos públicos. El tema de la migración es sumamente complejo y las experiencias de acogimiento son numerosas. Pero al existir compromisos entre los países para la defensa de los derechos humanos, la realidad hace que deban crearse nuevos lugares para la reflexión sobre los mecanismos de mejoramiento de los países.

\section{NUEVOS MECANISMOS DE INTERCAMBIOS}

Más allá de los numerosos intercambios existentes entre los países y los numerosos congresos realizados en los que participan miembros de la sociedad civil y expertos de varios orígenes, podemos mencionar algunas experiencias que han dado que hablar. 


\subsection{LA FUNDACIÓN EU-LAC}

Según informaciones del sitio web, fue creada por los Jefes de Estado y de Gobierno de la Unión Europea (UE) y de la Comunidad de Estados Latinoamericanos y Caribeños (CELAC) en 2010. Sus miembros son los países de la UE y CELAC y la propia UE. La Fundación es una herramienta de la asociación UE-CELAC y sus actividades nutren el diálogo intergubernamental, en línea con el Plan de Acción birregional.

La Fundación tiene la misión de fortalecer y promover la asociación estratégica birregional, mejorando su visibilidad y fomentando la participación activa de las sociedades civiles respectivas.

Durante la Reunión de Ministros de Relaciones Exteriores de la CELAC y la UE celebrada en Santo Domingo el 25 de octubre de 2016, se firmó el Acuerdo por el que se crea la Fundación como organización internacional. Se prevé la creación de una organización intergubernamental con sede en Hamburgo y que estará abierta a los países integrantes de ambas regiones. El texto constitutivo aún no fue ratificado para su entrada en vigor ${ }^{21}$.

Para su funcionamiento se prevé la creación de un Consejo Directivo formado por representantes de los Miembros de la Fundación UE-ALC que se reunirá a nivel de Altos Funcionarios y, en su caso, a nivel de Ministros de Asuntos Exteriores con motivo de las Cumbres UE-CELAC (artículo 9) previéndose la invitación a la Mesa Directiva de la Asamblea Parlamentaria EuroLatinoamericana (EuroLat) a designar a un representante de cada región como observadores en el Consejo Directivo.

El Consejo Directivo contará con dos presidentes: un representante de la UE y otro de los Estados Latinoamericanos y Caribeños y debe reunirse dos veces al año en sesión ordinaria. Estas reuniones deberán coincidir con las reuniones de Altos Funcionarios (SOM) de los Estados de la CELAC y de la UE (artículo 12). Será necesario contar con la presencia de más de la mitad de sus Miembros de cada región para las sesiones. Las decisiones se adoptarán por consenso de los Miembros presentes.

La Fundación se compondrá además de un Presidente encargado de representar a la Fundación en sus relaciones exteriores, garantizando una representación visible a través de contactos de alto nivel y de un Director Ejecutivo quien actúa como representante legal y en el cumplimiento de sus funciones no podrá solicitar ni recibir instrucciones de ningún Gobierno o de cualquier otro organismo. Ambos ocuparán sus cargos por cuatro años, renovables una vez y se elegirán respetando criterios de alternancia entre los bloques y previéndose que uno de los cargos sea ocupado por representante de cada región.

El artículo 16 prevé que las contribuciones se realizarán de manera voluntaria, sin perjuicio de la participación en el Consejo Directivo. 
El artículo 25 prevé que el acuerdo entre en vigor treinta días después de que ocho de las Partes de cada región, incluida la República Federal de Alemania y la UE, hayan depositado sus respectivos instrumentos de ratificación o adhesión ante el depositario, la Secretaría General del Consejo de la Unión Europea.

\subsection{EL FORO ACADEMICO PERMANENTE ALC-UE}

Se trata de una iniciativa de intercambios en materia de educación superior, ciencia, tecnología e innovación ${ }^{22}$. Se han realizado dos eventos en Santiago y Bruselas en 2013 y 2015, además de presentar informes sobre cooperación en materia de educación superior y la creación de un espacio común en estos ámbitos.

A la primera cita, según nos lo explica el diplomático chileno Héctor Casanueva ${ }^{23}$, acudieron 250 universidades y 600 académicos de ambas regiones y se sentaron las bases de un proceso de "Cumbres Académicas" organizadas por el Foro Académico Permanente (FAP ALC-UE). A este proceso se fueron integrando otras redes y asociaciones como el Consejo de Rectores de ALC-UE.

Se prevé la realización en paralelo a las cumbres oficiales de una Cumbre Empresarial, una Cumbre Social, una Cumbre Juvenil, y otra Sindical para intercambio de experiencia y elaboración de propuestas de la comunidad universitaria para avanzar en la creación del "Espacio Común de Educación Superior, Ciencia, Tecnología e Innovación ALC-UE”.

\subsection{ERASMUS MUNDUS SUD UE}

El Programa Erasmus nació como un lugar de intercambios para estudiantes en Europa. Sin embargo, el éxito hizo que trascienda las fronteras del continente y se expanda también a América latina. Así, sumándose a los programas de intercambios de cada país, se tiene un programa específico que, según el sitio web, es una Asociación entre 20 Universidades de América del Sur (Argentina, Bolivia, Brasil, Chile, Colombia, Ecuador, Paraguay, Perú, Uruguay y Venezuela) y Europa (Alemania, Eslovenia, España, Francia, Irlanda, Italia y Portugal) con el apoyo de instituciones asociadas, principalmente en América del Sur ${ }^{24}$

El programa es financiado por la Comisión Europea y sus principales objetivos son la promoción del espacio europeo de educación superior y el entendimiento intercultural mediante la cooperación con países de América del Sur, de acuerdo con los objetivos de política exterior de la UE para contribuir al desarrollo sostenible de América del Sur en el área de educación superior y a la vez mejorar e impulsar las perspectivas de carrera de los estudiantes.

\subsection{LA BUSQUEDA DE LA PAZ}


La Unión europea y la Organización de Estados Americanos tienen entre sus objetivos la solución pacífica de controversias. Más allá de que este objetivo sea un capítulo obligatorio en los programas de Derecho Internacional Público, debemos pensar en que la Unión europea, más allá de cualquier crítica que pueda hacérsele, ha logrado lo que ninguna organización anterior lo pudo hacer: evitar las guerras entre los miembros ${ }^{25}$.

Es frecuente escuchar en América que el ejemplo de integración más exitoso es el de la Unión europea. Sin dejar de lado los problemas que afectan al bloque, puede verse que el tándem franco-alemán dejó atrás siglos de conflictos y desconfianzas mutuas para firmar un tratado del Eliseo en 1963 que cambió la historia de las dos naciones ${ }^{26}$.

No solo se selló definitivamente la paz sino que se optó por realizar intercambios periódicos en todos los niveles y principalmente entre la juventud. A la fecha, en América los intercambios entre los países son aún escasos y creemos que deberían ser fomentados. Resulta claro que los jóvenes están llamados a no repetir los errores del pasado y solo conociendo la historia común y buscando una solución a los problemas existentes, podrá lograrse una verdadera integración. Hoy en día, las posibilidades de un conflicto armado en Europa son mínimas justamente porque existe ya una generación de personas que crecieron mirando a sus vecinos no como enemigos sino como asociados, a pesar de que subsisten diferencias de criterios y que las mismas seguirán existiendo, como es normal cuando se juntan dos o más personas.

¿Podríamos pensar en ejemplos más relevantes? Francamente, la cooperación y los intercambios en materias como protección de datos personales, asistencia judicial, seguridad jurídica y tantos aspectos del derecho resultan importantes. Por ello y como propuesta de cierre, queda abierta la reflexión sobre la cooperación en diversos ámbitos.

\section{PROPUESTAS DE CREACIÓN DE FOROS PERMANENTES PARA INTERCAMBIOS DE DIVERSAS EXPERIENCIAS}

La mención de los problemas comunes y de las iniciativas para su discusión efectuadas en este trabajo están lejos de ser las únicas. Sin embargo, podrá decirse que es difícil hacer un relevamiento de las mismas, incluso en épocas recientes, al no existir un lugar centralizado de información en diferentes áreas. Podemos hablar de Cumbres presidenciales, Congresos en determinadas áreas de la ciencia o encuentros de empresarios, pero será difícil encontrar un lugar común donde las personas, independientemente de sus países de residencia, puedan conocer las iniciativas que se realizan en una determinada materia.

Las dificultades económicas no permiten a los Estados promover los desplazamientos como quisieran, más allá que reconocen su utilidad. Difícilmente 
podrá discutirse que los intercambios de experiencias puedan resultar beneficiosos y que los mismos no deben realizarse solo a nivel institucional, siendo las universidades e institutos superiores otros ámbitos adecuados para tales intercambios.

Así, más allá de las vicisitudes políticas y de los problemas financieros, el uso de nuevas tecnologías podrá facilitar la comunicación de experiencias, resultados, vivencias dentro de las agendas de los organismos oficiales o privados, la actualidad y las necesidades de cada país y sociedad.

Con la sistematización de la información, podrá mostrarse la relevancia de los intercambios y experiencias entre los países UE-ALC que podrán incluso luego servir de experiencias a otras áreas.

\section{NOTAS}

1 Criterios de Copenhague: 1) la existencia de instituciones estables que garanticen la democracia, el Estado de derecho, el respeto de los derechos humanos y el respeto y la protección de las minorías; 2) la existencia de una economía de mercado en funcionamiento y la capacidad de hacer frente a la presión competitiva y las fuerzas del mercado dentro de la UE; 3) la capacidad para asumir las obligaciones que se derivan de la adhesión, incluida la capacidad para poner en práctica de manera eficaz las normas, estándares y políticas que forman el acervo comunitario, y aceptar los objetivos de la unión política, económica y monetaria. Fuente: Artículo 49 del Tratado de la UE. Adhesión UE. http://eur-lex.europa.eu/summary/glossary/accession_criteria_copenhague.html?locale=es

2 BREMMER, Ian: Curva J, Editorial El Ateneo, 2007, 384 p.

3 JAUREGUI, Ramón: “Una cumbre para el futuro”, El País, Madrid, 29 oct. 2016. https://elpais. com/internacional/2016/10/28/actualidad/1477607112_035337.html Consultado el 20 de enero de 2018

4 Tratado de Chaguaramas. http://caricom.org/the-caribbean-court-of-justice

5 Sitio oficial de la Corte: http://www.ccj.org/. EFE: Ecuador impulsa en UNASUR la creación de Corte penal suramericana, 16 de setiembre de 2016. https://www.efe.com/efe/america/politica/ ecuador-impulsa-en-la-unasur-creacion-de-corte-penal-suramericana/20000035-3040416 Consultado el 20 de enero de 2018

6 BUTKEVICIENE, Jolita: Experiencias de la Unión Europea con América Latina, Euroclima, 2017. http://www.euroclima.org/es/paises/item/1344-experiencias-de-la-union-europea-con-americalatina Consultado el 20 de diciembre de 2017

7 DW: UE-América latina, nuevo marco para las relaciones. http://www.dw.com/es/ueam\%C3\%A9rica-latina-nuevo-marco-para-las-relaciones/a-39649313

8 Asistencia consular. Directiva (UE) 2015/637 http://ec.europa.eu/justice/citizen/files/2015_637_ es.pdf

9 http://www.comunidadandina.org/Prensa.aspx?id=3349\&accion=detalle\&

10 Acuerdo interinstitucional de cooperación en materia de asistencia consular https:// alianzapacifico.net $/$ ?wpdmdl $=4397$

11 Carta de Bogotá http://www.oas.org/es/sla/ddi/tratados_multilaterales_interamericanos_A-41_ carta_OEA.asp 
12 http://www.echr.coe.int/Documents/Convention_SPA.pdf

13 Adoptada en Nueva York, Estados Unidos, el 28 de septiembre de 1954 por una Conferencia de Plenipotenciarios convocada por el Consejo Económico y Social en su resolución 526 A (XVII), de 26 abril de 1954. http://www.acnur.org/fileadmin/scripts/doc.php?file=fileadmin/Documentos/ BDL/2001/0006 Consultado el 20 de enero de 2018

14 Adoptada en Nueva York, Estados Unidos, el 30 de agosto de 1961 por una Conferencia de Plenipotenciarios que se reunió en 1959 y nuevamente en 1961, en cumplimiento de la resolución 896 (IX) de la Asamblea General, de 4 de diciembre de 1954. http://www.acnur.org/fileadmin/ scripts/doc.php?file=fileadmin/Documentos/BDL/2001/0007 Consultado el 20 de enero de 2018

15 ACNUR: Campaña de las convenciones sobre Apatridia, 2017. http://www.acnur.org/a-quienayuda/apatridas/campana-de-las-convenciones-sobre-la-apatridia/ Consultado el 20 de enero de 2018

16 POLETTI ADORNO, Alberto: "Manual del migrante paraguayo", Intercontinental Editora, Asunción, 2012, p. 214

17 Convenio sobre nacionalidad del 6 de noviembre de 1997. http://www.migrarconderechos.es/ legislationMastertable/legislacion/Convenio_nacionalidad_06_11_1997

18 Constitución de Argentina http://leyes-ar.com/constitucion_nacional/25.htm

19 OSPINA VALENCIA, José: Migración ilegal en América Latina, explotación y negocio, DW, 24 de julio de 2017. http://www.dw.com/es/migraci\%C3\%B3n-ilegal-en-am\%C3\%A9rica-latinaexplotaci\%C3\%B3n-y-negocio/a-39820243

20 BBC, Las rutas más peligrosas de la migración ilegal en América Latina, 22 de abril de 2015. http://www.bbc.com/mundo/noticias/2015/04/150422_rutas_inmigracion_latinoamerica_ep. Más allá de la migración hacia Estados Unidos, se han desarrollado otras vías como por ejemplo, la migración de haitianos a Brasil a través del Estado de Acre. BBC: "El coyote, nuevo personaje en la frontera de Brasil, 13 de abril de 2013. http://www.bbc.com/mundo/noticias/2013/04/130418_ brasil_inmigracion_indocumentados_coyotesnm

21 Acuerdo por el que se crea la Fundación Internacional UE-ALC. http://www.itamaraty.gov.br/ images/ed_integracao/docs_CELAC/EULAC.2016ESP.pdf

22 Foro Académico Permanente ALC-UE http://www.fap-alc-ue.com/

23 CASANUEVA, Héctor: “Un 'pilar académico' para la asociación de América latina, el Caribe y la Unión europea, América Economía, 24 de agosto de 2017. https://www.americaeconomia.com/ analisis-opinion/un-pilar-academico-para-la-asociacion-de-latina-el-caribe-y-la-union-europea

24 Erasmus Mundus Sud UE http://sudue.eu/es/

25 POLETTI AdORNO, Alberto: "Un Nobel de la Paz para la Unión Europea”, Revista de la Academia Diplomática y Consular "Carlos Antonio López", Ministerio de Relaciones Exteriores de la República del Paraguay, No 2, 2013, p. 79-91. http://www.mre.gov.py/V1/Adjuntos/revista_ diplomatica_n_2.pdf

$26 \mathrm{http}: / /$ tratadoeliseo.tumblr.com 


\section{Bibliografía}

ALCAIDE FERNÁNDEZ, Joaquín y CASADO RAIGÓN, Rafael (coord.): Curso de Derecho de la Unión Europea, 2a ed., Editorial Tecnos, 2014.

ALL, Paula; OVIEDO ALBAN, Jorge y VESCOVI, Eduardo (coord.): La actividad internacional de la empresa, Editorial Ibáñez, Bogotá, ASADIP, 2017

BREMMER, Ian: Curva J, Editorial El Ateneo, 2007, 384 p.

GUIMARAES CHAI, Cássius, COELHO DE ZEVEDO, Elda y CHAVES MESQUITA, Valena (coord): "Ensaios críticos: do político ao jurídico", Serie Teoría Política e sistemas de justiça, Vol. 1, Campos dos Goytacazes, RJ, Brasil Multicultural, 2016, ISBN 978-855635-023-7. http://www.brasilmulticultural.com.br/_imagens/Livro_TPSJ-v-01.pdf

GUIMARAES CHAI, Cássius, COELHO DE ZEVEDO, Elda y CHAVES MESQUITA, Valena (coord): "Direito, trabalho e desconhecimento: desafios contra os retrocessos em direitos humanos”, Serie Teoria Política e sistemas de justiça, Vol. 2, Campos dos Goytacazes, RJ, Brasil Multicultural, 2016, ISBN 978-85-5635-024-4. http://www. brasilmulticultural.com.br/_imagens/Livro_TPSJ-v-02.pdf

HINOJOSA MARTÍNEZ, Luis Miguel: El reparto de competencias entre la Unión Europea y sus estados miembros. Valencia, Tirant lo Blanch, 2006. 397 p.

MORENO RODRIGUEZ, Diego: Los perfiles de la justicia constitucional en el ordenamiento jurídico paraguayo, (La Ley Paraguaya, 2005), p. 154-185

MORENO RODRIGUEZ, José Antonio: La Contratación Internacional y los Procesos de Integración Regional, Revista de la Facultad de Derecho de la Universidad Nacional de Asunción, 2006

MORENO RODRIGUEZ, José Antonio: La Convención más trascendente en la historia del Derecho Privado, Revista Jurídica La Ley Paraguaya, Año 2, No. 52, 2009 Derecho Aplicable a la Contratación y el Arbitraje Internacional, Revista Jurídica Universidad Católica de Asunción, 2011

MORENO RODRIGUEZ, José Antonio: Autonomía contractual transfronteriza, en el Libro homenaje a Roberto Ruíz Díaz Labrano, CEDEP, 2013

OPPENHEIMER, Andrés: Basta de historias. La obsesión latinoamericana con el pasado y las 12 claves del futuro, Random House Mondadori, De bolsillo, Buenos Aires, 2010, $522 \mathrm{p}$.

PASTROZA, Paulina (dir), Programa de Estudios de Europeos, Tensiones en Europa: desafíos para la integración, RIL Editores, Concepción, Chile, 2013

POLETTI ADORNO, Alberto: “Derecho constitucional comparado”, Intercontinental Editora, Asunción, 2011 
POLETTI ADORNO, Alberto: "Manual del migrante paraguayo", Intercontinental Editora, Asunción, 2012

POLETTI ADORNO, Alberto: “Un Nobel de la Paz para la Unión Europea”, Revista de la Academia Diplomática y Consular "Carlos Antonio López”, Ministerio de Relaciones Exteriores de la República del Paraguay, № 2, 2013, p. 79-91. Internet: http://www.mre. gov.py/V1/Adjuntos/revista_diplomatica_n_2.pdf

REVIRIEGO, Fernando y AGUIAR DE LUQUE, Luis: Implicancias constitucionales de giro político en algunos países Iberoamericanos (2005/2007), Cuadernos de derecho público, ISSN 1138-2848, No 27, 2006, págs. 227-293

RUIZ DIAZ LABRANO, Roberto: “Mercosur, Integración y Derecho”, Ediciones Ciudad Argentina, Buenos Aires, e Intercontinental Editora, Asunción, Año 1998.

SUÑE, Natasha: Principios de organización administrativa en la estructura institucional del MERCOSUR, Revista digital de derecho administrativo, Universidad del Externado de Colombia, Núm. 13 (2015). http://revistas.uexternado.edu.co/index.php/Deradm/ article/view/4192/4815 


\section{Resumen}

La cooperación UE-LAC resulta fructífera en varios ámbitos y es beneficiosa para los países. Por ende, más allá de presentarse algunos elementos de cooperación en el ámbito jurídico, se insiste en la necesidad de crear nuevos foros de intercambios, principalmente en el ámbito juvenil. Los problemas conjuntos tales como el cambio climático, la lucha contra el terrorismo y la promoción del Estado de derecho requieren no solo contactos a nivel gubernamental sino también a nivel de la sociedad civil y sin duda, la creación de espacios de intercambio permanentes facilitará la obtención de objetivos comunes.

Palabras claves: América Latina - Caribe - Unión europea - Cooperación internacional - Estado de Derecho - Terrorismo - Cambio climático - Migraciones - Juventud 


\begin{abstract}
Cooperation between EU-LAC is fruitful in several areas and is beneficial for countries. Therefore, beyond presenting some elements of cooperation in the legal field, it insists on the need to create new exchange forums, mainly in the youth field. Joint problems such as climate change, the fight against terrorism and the promotion of the rule of law require not only contacts at the governmental level but also at the level of civil society and, without a doubt, the creation of permanent exchange spaces will facilitate the pursuit of common objectives.
\end{abstract}

Keywords: Latin America - Caribbean Zone - European Union - International cooperation - Rule of law - Terrorism - Climate change - Migrations - Youth 


\section{Resumo}

A cooperação UE-ALC é frutífera em várias áreas e é benéfica para os países. Portanto, além de apresentar alguns elementos de cooperação no campo jurídico, insiste na necessidade de criar novos fóruns de intercâmbio, principalmente no campo da juventude. Problemas comuns como a mudança climática, a luta contra o terrorismo e a promoção do Estado de Direito requerem não só contatos no nível governamental, mas também ao nível da sociedade civil e, sem dúvida, a criação de espaços de intercâmbio permanente facilitará a obtenção de objetivos comuns.

Palabras-chave: América latina - Caribe - União europeia - Cooperação internacional Estado de direito - Terrorismo - Cambio climático - Migrações - Juventude 